\title{
Metal azides under pressure: An emerging class of high energy density materials
}

\author{
G VAITHEESWARAN* and K RAMESH BABU \\ Advanced Centre of Research in High Energy Materials (ACRHEM), University of Hyderabad, \\ Prof. C R Rao Road, Gachibowli, Hyderabad 500 046, India \\ e-mail:gvsp@uohyd.ernet.in
}

\begin{abstract}
Metal azides are well-known for their explosive properties such as detonation or deflagration. As chemically pure sources of nitrogen, alkali metal azides under high pressure have the ability to form polymeric nitrogen, an ultimate green high energy density material with energy density three times greater than that of known high energetic materials. With this motive, in this present work, we try to address the high-pressure behaviour of $\mathrm{LiN}_{3}$ and $\mathrm{KN}_{3}$ by means of density functional calculations. All the calculations are performed with the inclusion of van der Waals interactions at semi empirical level, as these materials are typical molecular solids. We found that both $\mathrm{LiN}_{3}$ and $\mathrm{KN}_{3}$ are structurally stable up to the studied pressure range of $60 \mathrm{GPa}$ and $16 \mathrm{GPa}$, respectively. At ambient conditions both the materials are insulators with a gap of $3.48 \mathrm{eV}(\mathrm{LiN})_{3}$ and $4.08 \mathrm{eV}\left(\mathrm{KN}_{3}\right)$ and as pressure increases the band gap decreases and show semiconducting nature at high pressures. We also found that the compressibility of both the crystals is anisotropic which is in good agreement with experiment. Our theoretical study proved that the materials under study may have the ability to form polymeric nitrogen because of the decrease in interazide ion distance and possible overlapping of $\mathrm{N}$ atomic orbitals.
\end{abstract}

Keywords. Energetic materials; density functional theory; band gap; compressibility.

\section{Introduction}

Inorganic azides are interesting class of energetic compounds exhibiting a wide variety of physical and chemical properties. ${ }^{1}$ These materials belong to the category of primary explosives and can be used to detonate the secondary explosives. ${ }^{2}$ Among the inorganic metal azides, monovalent azides such as alkali metal azides are chemically and structurally simple compounds and have wide range of practical importance as explosives, gas generators, and also photographic materials at low temperatures. ${ }^{3}$ Alkali metal azides are ionic molecular crystals having layered structure with alternating planes of azide ion and metal ions. ${ }^{4}$ Among these alkali metal azides both $\mathrm{LiN}_{3}$ and $\mathrm{NaN}_{3}$ crystallize in monoclinic structure with space group of $\mathrm{C} 2 / \mathrm{m}^{5}$ and the remaining azides such as $\mathrm{KN}_{3}, \mathrm{RbN}_{3}$ and $\mathrm{CsN}_{3}$ crystallize in tetragonal structure with space group of $\mathrm{I} 4 / \mathrm{mcm}{ }^{6}$ Recently this class of metal azides received much interest from the high energy materials research community because of their ability to form the polymeric networks of single bonded nitrogen, which is considered to be a high energy density material with energy density nearly three times greater than those of conventional high explosive materials known today. 7,8

*For correspondence
By using sodium azide as starting material, Eremets et al. ${ }^{9}$ reported the formation of polymeric nitrogen by combined high pressure X-ray powder diffraction and Raman spectroscopy techniques. Their study concluded that double bonded $\mathrm{N}$ atoms of the azide ion become single bonded $\mathrm{N}$ atoms at a pressure of $120 \mathrm{GPa}$. Medvedev et al. ${ }^{10}$ reported experimentally that $\mathrm{LiN}_{3}$, which is iso-structural to $\mathrm{NaN}_{3}$, has the capability of forming polymeric networks beyond the compression of $60 \mathrm{GPa}$. Recently the compression experiments on iso-structural heavier alkali metal azides $\mathrm{CsN}_{3}$ and $\mathrm{KN}_{3}$ have been performed. ${ }^{11,12}$ The high pressure experiment on $\mathrm{CsN}_{3}$ reveals that the system undergoes a sequence of phase transitions starting from ambient tetragonal phase to high pressure trigonal structure. ${ }^{11}$ In the case of $\mathrm{KN}_{3}$ the high pressure experiments were carried out up to the pressure of $37 \mathrm{GPa}$ and found that the system may undergo structural phase transition at around 15.5 GPa. However, the high pressure structural phase could not be identified by the authors. ${ }^{12}$

From theoretical perspective, recently we have studied the high pressure behaviour of $\mathrm{LiN}_{3}$ using density functional theory without including the van der Waals (vdW) interactions. ${ }^{13}$ The calculated equilibrium volumes are slightly overestimated compared with the experiment, which can be attributed to the lack of inclusion of vdW interactions in the calculations. The study 
also revealed that the monoclinic $\mathrm{LiN}_{3}$ is stable up to the studied pressure range of $60 \mathrm{GPa}$, which is in good agreement with experiment. In the case of $\mathrm{KN}_{3}$, there is no first principles study available in explaining the high pressure behaviour of the system with and without including vdW interactions. Hence in this present work, we aim to study the high pressure behaviour of monoclinic $\mathrm{LiN}_{3}$ and tetragonal $\mathrm{KN}_{3}$ using first principles density functional theory calculations including $\mathrm{vdW}$ interactions. In particular, we aim to explain the structural properties, electronic band structure and bonding properties of the azides and the changes brought in them by the application of pressure as they are very important in understanding the formation of polymeric nitrogen. The remainder of the paper is organized as follows: the next section deals with the computational details of the present study and in section 3, we have presented the results and discussion. Finally, we have given brief conclusions.

\section{Computational details}

All the calculations are performed with plane wave pseudo potential density functional computer code Cambridge Serial Total Energy Package (CASTEP). ${ }^{14}$ The generalized gradient approximation (GGA) using Perdew-Burke-Ernzerhof (PBE) parameterization was used for exchange-correlation. ${ }^{15}$ As the materials under study are layered structure crystals, where vdW interactions play a major role in crystal binding and the physical and chemical properties, we used semi empirical dispersion correction (DFT+D) proposed by Grimme within PBE parameterization to treat vdW interactions. ${ }^{16}$ For electron-ion interactions, we have used Vanderbilt type ultrasoft pseudo potentials. ${ }^{17}$ The pseudo potentials of $\mathrm{Li} 2 \mathrm{~s}^{1}, \mathrm{~N} 2 \mathrm{~s}^{2} 2 \mathrm{p}^{3}$ for $\mathrm{LiN}_{3}$ and $\mathrm{K}$ $3 \mathrm{~s}^{2} 3 \mathrm{p}^{6} 4 \mathrm{~s}^{1}$ are used for the study. The plane wave cut-off energy of $520 \mathrm{eV}$ is used. The k-point mesh is created by Monkhorst-Pack grid scheme ${ }^{18}$ using $5 \times 8 \times 5$ for both $\mathrm{LiN}_{3}$ and $\mathrm{KN}_{3}$, respectively. The total energy convergence for these parameters was checked and found that the error is less than $1 \mathrm{meV}$ per atom. The selfconsistent convergence of the total energy is found to be $5 \times 10^{-7} \mathrm{eV} /$ atom and the maximum force on the atom is found to be $10^{-4} \mathrm{eV} / \AA$. The experimental crystal structures given by Pringle ${ }^{5}$ for $\mathrm{LiN}_{3}$ and for $\mathrm{KN}_{3}$ by Muller ${ }^{6}$ are taken as the starting input for the calculations.

\section{Results and discussion}

\subsection{Structural properties of $\mathrm{LiN}_{3}$ and $\mathrm{KN}_{3}$ at ambient pressure}

For all theoretical calculations, it is necessary to obtain the equilibrium crystal structure. Therefore to determine the theoretical equilibrium crystal structure, we have performed full structural optimization of monoclinic $\mathrm{LiN}_{3}$ and tetragonal $\mathrm{KN}_{3}$ within DFT (PBE) and $\mathrm{DFT}+\mathrm{D}(\mathrm{PBE}+\mathrm{G} 06)$ methods. The optimized crystal structures and the corresponding reciprocal lattices with the Brillouin zones are shown in figure $1 \mathrm{a}$ and $\mathrm{b}$ for

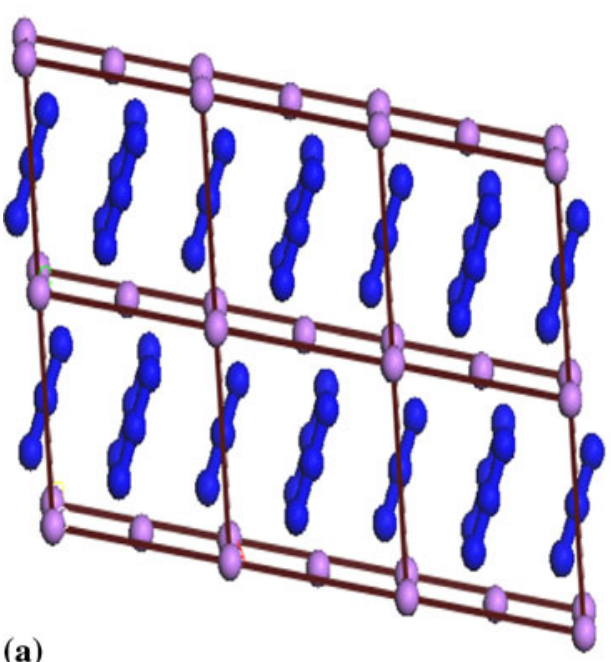

(a)

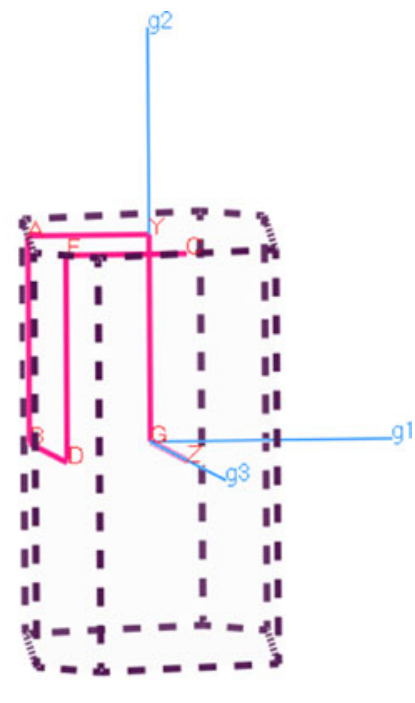

(b)

Figure 1. (a) The optimized crystal structure (violet ball denotes ' $\mathrm{Li}$ ' atom and blue ball denotes ' $N$ ' atom) and (b) reciprocal lattice of monoclinic $\mathrm{LiN}_{3}$. 


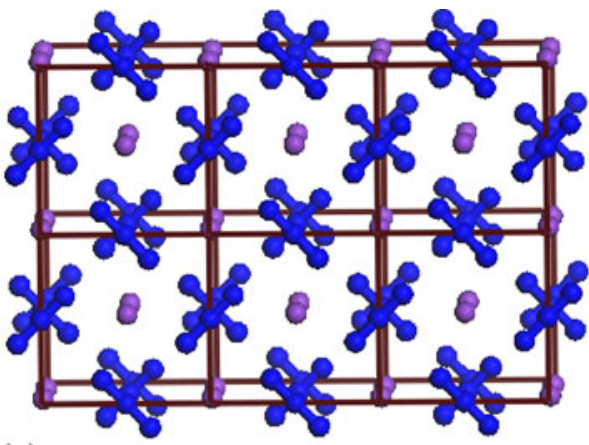

(a)

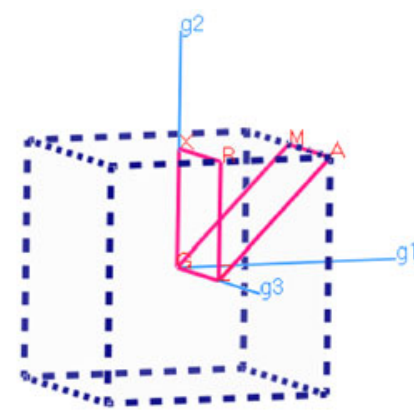

(b)

Figure 2. (a) The optimized crystal structure (violet ball denotes ' $K$ ' atom and blue ball denotes ' $\mathrm{N}$ ' atom) and (b) reciprocal lattice of tetragonal $\mathrm{KN}_{3}$.

$\mathrm{LiN}_{3}$ and for $\mathrm{KN}_{3}$ figure $2 \mathrm{a}$ and $\mathrm{b}$. The calculated lattice constants and the atomic fractional co-ordinates of mid and end $\mathrm{N}$ atoms are presented in table 1. Our calculated ground state parameters are in good comparison with earlier $a b$-initio studies on $\mathrm{LiN}_{3} .{ }^{19,20}$ For both the compounds, the values obtained through DFT (PBE) are over estimated from that of the experimental values. This trend of over estimation of lattice parameters is quite common for the molecular crystals of the present type where vdW interactions play a dominant role in crystal binding which cannot be dealt with the general DFT-GGA-PBE functional. From table 1, we can also clearly see that the lattice parameters obtained through the $\mathrm{DFT}+\mathrm{D}$ method using $\mathrm{PBE}+\mathrm{G} 06$ functional is very close to the experimental data with error less than $1 \%$ for both the compounds. In particular the calculated lattice parameter ' $a$ ' within $D F T+D$ is in very good agreement with experiment indicating that the $\mathrm{vdW}$ interactions are strong along the a-axis in both the compounds over the other lattice axes.

\subsection{Structural properties of $\mathrm{LiN}_{3}$ and $\mathrm{KN}_{3}$ under high pressure}

In order to study the pressure effect on $\mathrm{LiN}_{3}$ and $\mathrm{KN}_{3}$ systems, we have taken the ambient optimized crystal structure and applied hydrostatic pressure of $60 \mathrm{GPa}$ on monoclinic $\mathrm{LiN}_{3}$ system with successive intervals of $5 \mathrm{GPa}$ and $16 \mathrm{GPa}$ to the tetragonal $\mathrm{KN}_{3}$ system with successive intervals of $2 \mathrm{GPa}$, respectively. The calculated equation of state (EOS) of both $\mathrm{LiN}_{3}$ and $\mathrm{KN}_{3}$ are compared with available experimental data ${ }^{10,12}$ in figure $3 \mathrm{a}$ and $\mathrm{b}$. For both the compounds the volume compression upon pressure with DFT $+\mathrm{D}$ method is found to be in excellent agreement with experimental volume reduction. In particular, the calculated EOS of $\mathrm{KN}_{3}$ with DFT+D is in excellent agreement with experiment over the entire studied pressure range. This clearly shows that DFT+D describe the $\mathrm{KN}_{3}$ system accurately than that of usual DFT. Over the studied pressure range of both compounds, we could not

Table 1. The calculated ground state structural properties of $\mathrm{LiN}_{3}$ and $\mathrm{KN}_{3}$ within DFT and DFT+D. For $\mathrm{LiN}_{3}$ the calculated volume is per primitive cell, whereas for $\mathrm{KN}_{3}$ it is per unit cell.

\begin{tabular}{llllllll}
\hline Method & $a(\AA)$ & $b(\AA)$ & $c(\AA)$ & $\beta\left(^{0}\right)$ & $\mathrm{V}\left(\AA^{3}\right)$ & Mid N & End N \\
\hline & \multicolumn{7}{c}{$\mathrm{LiN}_{3}$} \\
DFT (GGA-PBE) & 5.7611 & 3.376 & 5.094 & 108.6 & 46.9 & 0.1005 & 0.7490 \\
DFT+D(GGA-PBE+G06) & 5.6857 & 3.219 & 4.8844 & 102.7 & 43.6 & 0.1044 & 0.7448 \\
Expt $^{\mathrm{b}}$ & 5.627 & 3.319 & 4.979 & 107.4 & 44.4 & 0.1048 & 0.7357 \\
& \multicolumn{7}{c}{$\mathrm{KN}_{3}$} \\
DFT (GGA-PBE) & 6.205 & 6.205 & 7.207 & & 277.5 & 0.1388 & 0.6388 \\
DFT+D(GGA-PBE+G06) & 6.1015 & 6.1015 & 6.9615 & & 259.2 & 0.1411 & 0.6411 \\
Expt $^{\mathrm{c}}$ & 6.113 & 6.113 & 7.094 & 265.1 & 0.1358 & 0.6358 \\
\hline
\end{tabular}

${ }^{\mathrm{a}} \operatorname{Ref}{ }^{13},{ }^{\mathrm{b}} \operatorname{Ref}^{5},{ }^{\mathrm{c}} \operatorname{Ref}{ }^{6}$ 

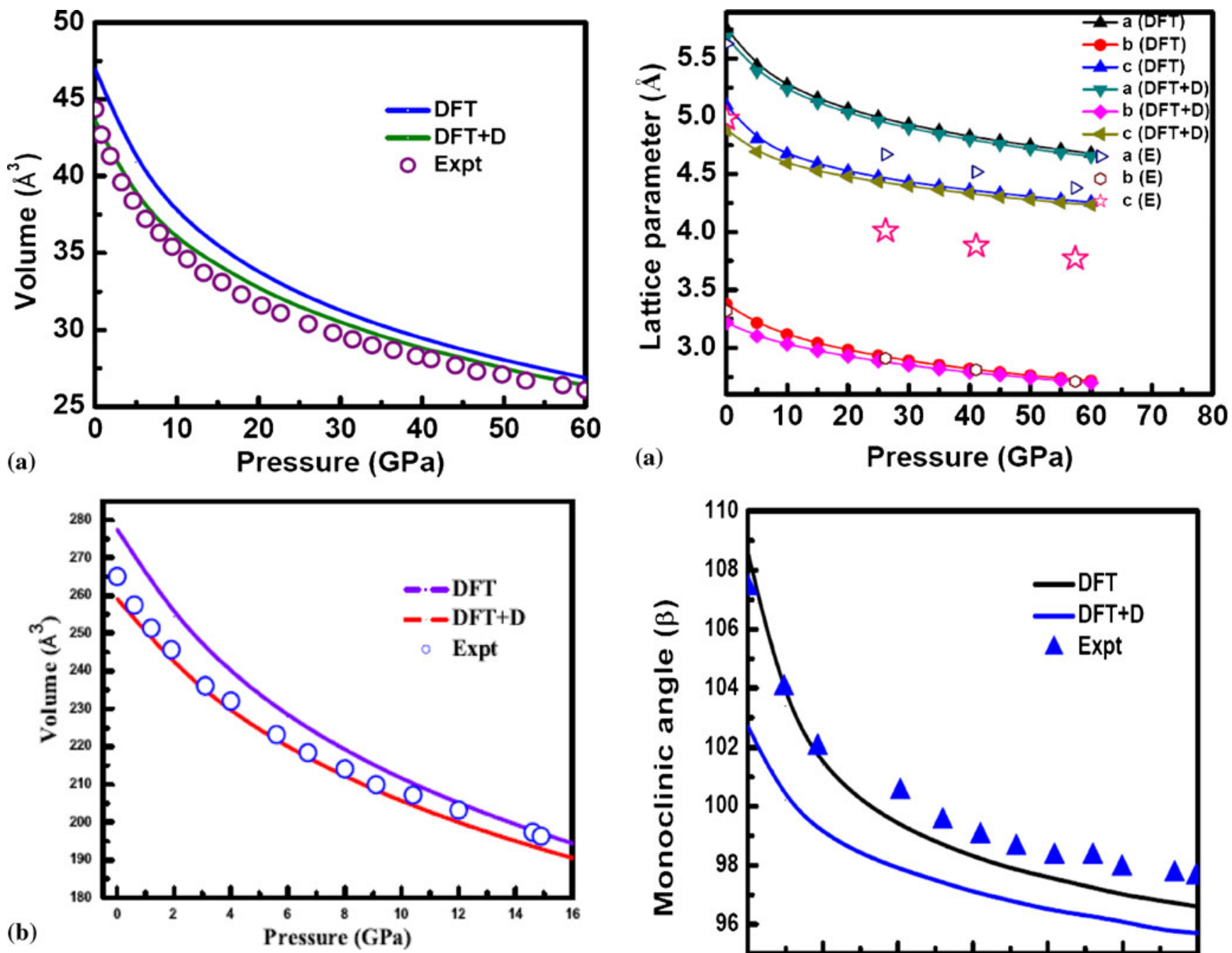

Figure 3. Pressure dependence of volume of (a) $\mathrm{LiN}_{3}$ and (b) $\mathrm{KN}_{3}$.

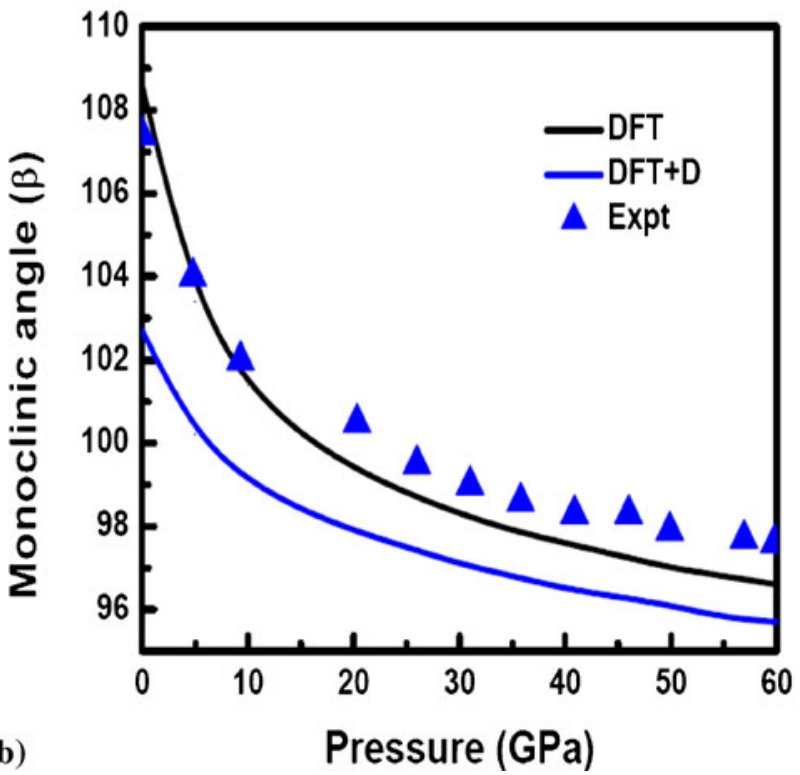

Figure 4. (a) Pressure dependence of lattice parameters of $\mathrm{LiN}_{3}$ and (b) pressure dependence of monoclinic angle $(\beta)$ of $\mathrm{LiN}_{3}$.

cating that both the systems are structurally stable with no phase transition. In figure $4 \mathrm{a}$ we have shown the pressure dependence of lattice parameters of $\mathrm{LiN}_{3}$. Qualitatively our calculations reproduce the trend of reduction of lattice parameters upon pressure. The deviation from the experimental data was found to be more for the c-axis with both the DFT and DFT+D methods. The reason for this behaviour might be due to the fact that error in our ambient pressure value of ' $c$ ' lattice parameter is more compared to that of the other axis. The pressure dependence of lattice parameter ' $b$ ' is found to be very good agreement with our calculations using DFT+D with error less than $0.5 \%$ over the entire pressure range. In figure $4 \mathrm{~b}$ we have shown the pressure dependence of monoclinic angle $\beta$ in comparison with experiment. We can see that the error is quite lower with DFT than that of DFT+D. Overall, our high pressure study on $\mathrm{LiN}_{3}$ suggests that the compressibility

of $\mathrm{LiN}_{3}$ is anisotropic as the lattice parameters have different compression behaviour with pressure, which is also consistent with experiment. ${ }^{10}$ The pressure dependence of lattice parameters of $\mathrm{KN}_{3}$ along with experimental data is shown in figure 5. Clearly the lattice parameter ' $a$ ' calculated with DFT $+\mathrm{D}$ is found to be in close agreement with experiment when compared to $\mathrm{c}$-axis. The reduction in lattice parameters shows that the compressibility of $\mathrm{KN}_{3}$ crystal is also anisotropic as expected in experiment. ${ }^{12}$

\subsection{Electronic structure of $\mathrm{LiN}_{3}$ and $\mathrm{KN}_{3}$}

The knowledge of band structure is quite important in understanding the electronic properties of the materials. In this section, we explain the calculated electronic 


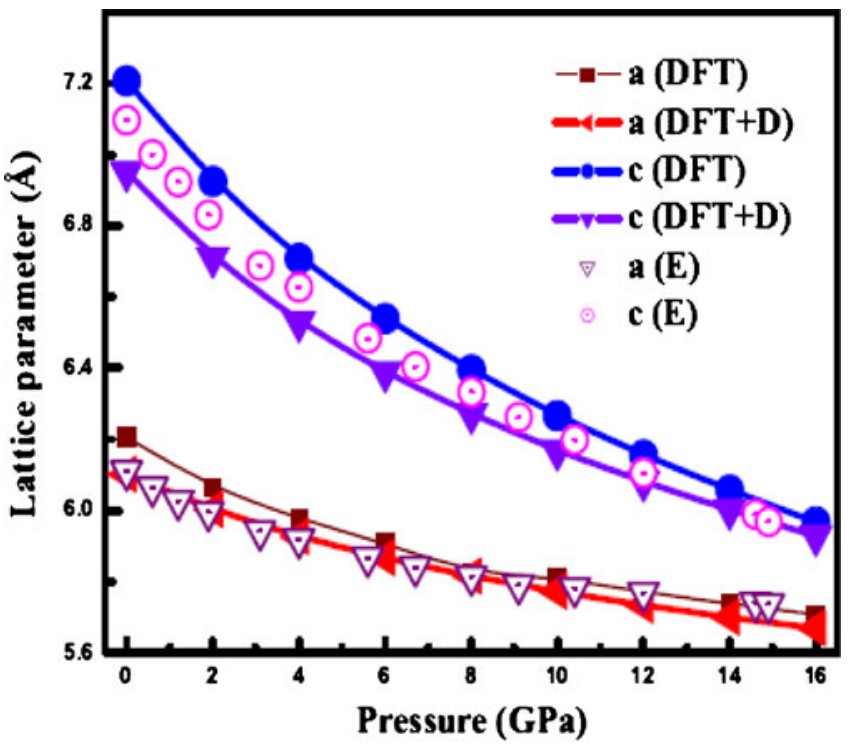

Figure 5. Pressure dependence of lattice parameters of $\mathrm{KN}_{3}$.

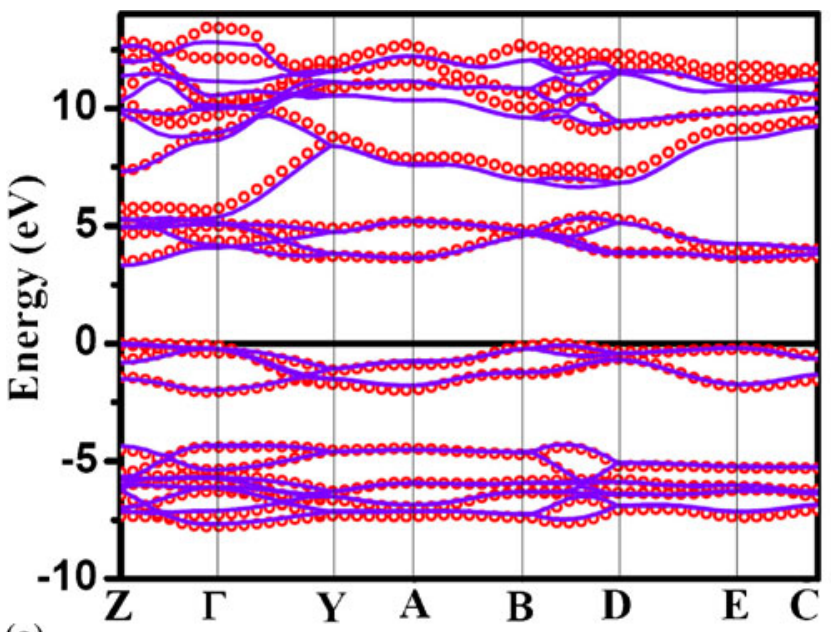

(a)

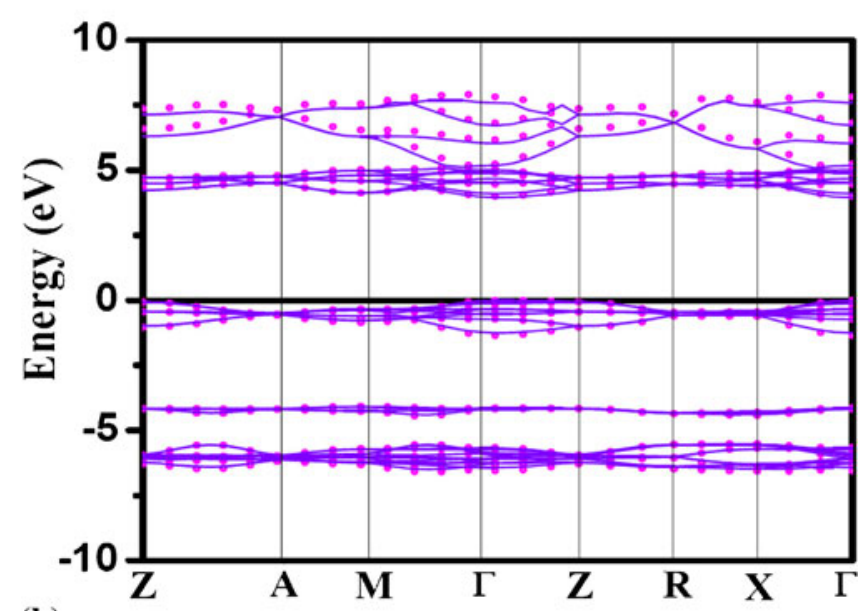

(b)

Figure 6. (a) Electronic band structure of $\mathrm{LiN}_{3}$ calculated at ambient pressure and (b) electronic band structure of $\mathrm{KN}_{3}$ at ambient pressure calculated using DFT (solid lines) and $\mathrm{DFT}+\mathrm{D}$ (circles). band structures of the azides $\mathrm{LiN}_{3}$ and $\mathrm{KN}_{3}$ using both the methods DFT and DFT + D. At ambient pressure, the calculated band structures of $\mathrm{LiN}_{3}$ and $\mathrm{KN}_{3}$ along the high symmetry points in the Brillouin zone are shown in figure $6 \mathrm{a}$ and $\mathrm{b}$ where we compare the results of DFT and DFT+D. Clearly, both the compounds are having indirect band gap with valence band maximum (VBM) located at $\mathrm{Z}$ and conduction band minimum (CBM) located at $\mathrm{Z}$ in $\mathrm{LiN}_{3}$ and for $\mathrm{KN}_{3}$ it is between the $\Gamma(\mathrm{VBM})-\Gamma(\mathrm{CBM})$. The band gap values of $\mathrm{LiN}_{3}$ is $3.32 \mathrm{eV}$ within DFT and $3.48 \mathrm{eV}$ using $\mathrm{DFT}+\mathrm{D}$, whereas for $\mathrm{KN}_{3}$ the values are $4.06 \mathrm{eV}$ (DFT) and $4.08 \mathrm{eV}(\mathrm{DFT}+\mathrm{D})$, respectively indicating both the compounds to be direct band gap insulators. The calculated band gaps within DFT $+\mathrm{D}$ are slightly larger than that of DFT values. However, we expect that our calculated band gaps might be underestimated from the experimental data, which is a common feature in all DFT and DFT + D calculations. ${ }^{21}$ Unfortunately, there

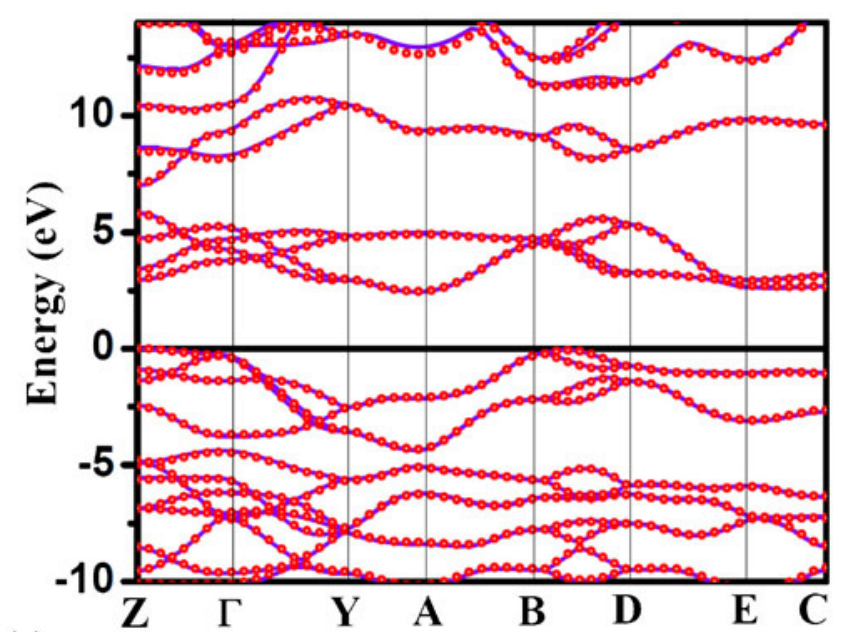

(a)

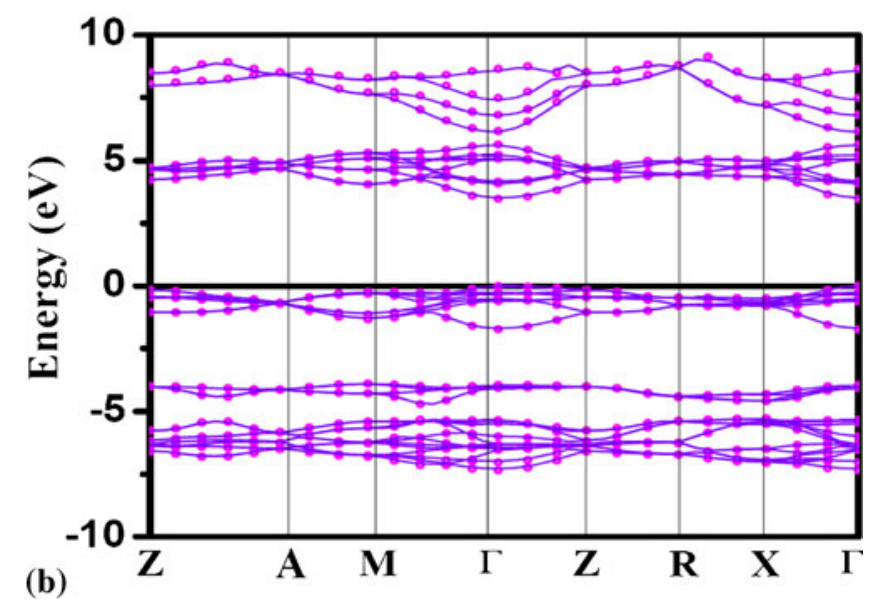

Figure 7. (a) Electronic band structure of $\mathrm{LiN}_{3}$ calculated at pressure of $60 \mathrm{GPa}$ and (b) electronic band structure of $\mathrm{KN}_{3}$ at pressure of $16 \mathrm{GPa}$ using DFT (solid lines) and $\mathrm{DFT}+\mathrm{D}$ (circles). 
are no experimental studies available to estimate the percentage of deviation regarding the calculated band gap values. We hope our calculations will stimulate experimentalists in this direction.

If we look at the calculated band structure of $\mathrm{LiN}_{3}$ with DFT and DFT+D as shown in figure 6a, the magnitude of the DFT + D corrections brought to the band energies varies for different bands. In particular, the inclusion of the dispersion corrections to the band energies in the valence band are more for $\mathrm{N}-\mathrm{p}$ bands that are farther from the Fermi level, which is the VBM. The corrections are even more for N-p bands that are situated at the energies greater than $5 \mathrm{eV}$ in the conduction band. Overall, the inclusion of dispersion corrections improves significantly the band energies both in valence and conduction bands. In the case of electronic band structure of $\mathrm{KN}_{3}$ as shown in figure $6 \mathrm{~b}$, we also observe the same features. In figure $7 \mathrm{a}$ and $\mathrm{b}$, we have shown the band structures of $\mathrm{LiN}_{3}$ and $\mathrm{KN}_{3}$ at $60 \mathrm{GPa}$ and
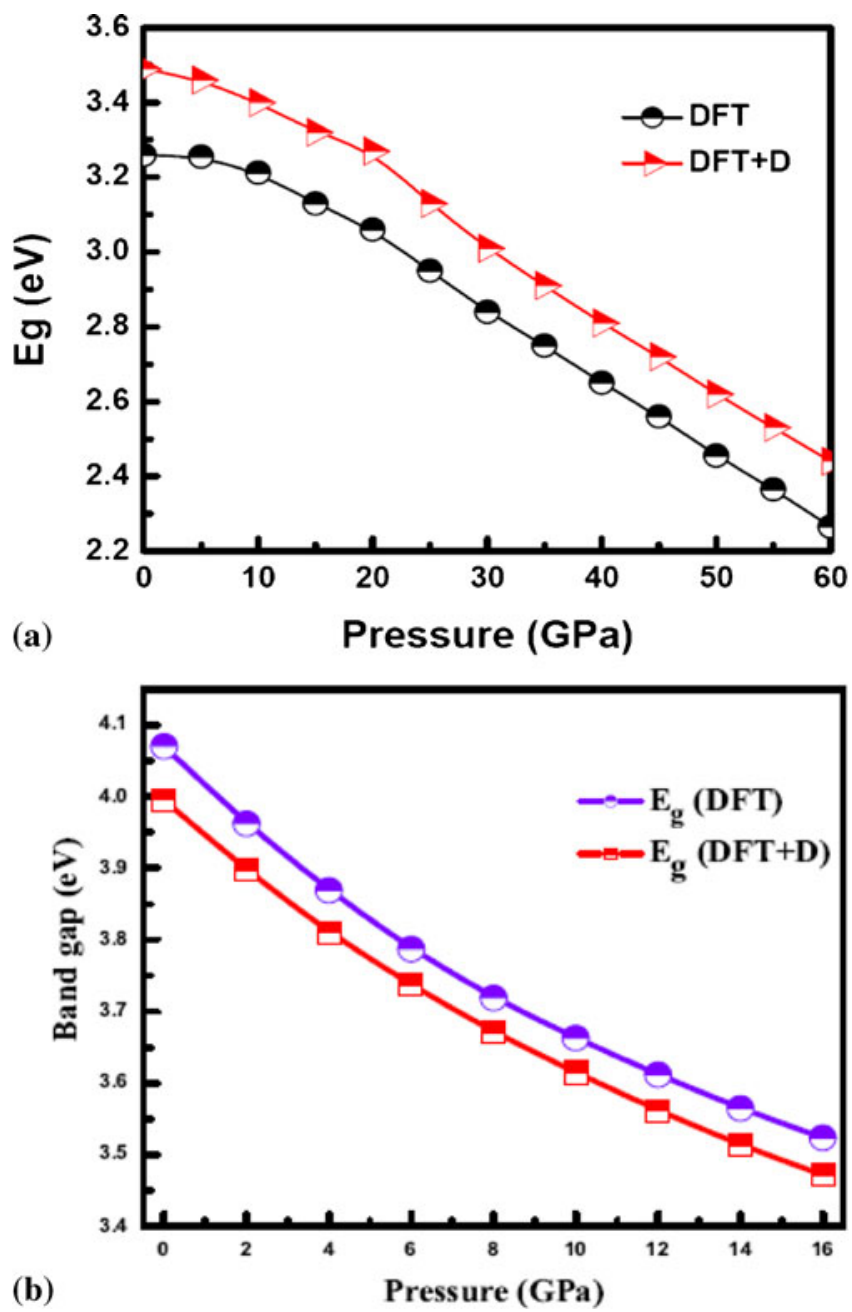

Figure 8. (a) The pressure dependence of electronic band gap of $\mathrm{LiN}_{3}$ and (b) the pressure dependence of electronic band gap of $\mathrm{KN}_{3}$.
$16 \mathrm{GPa}$, respectively. Clearly, under pressure the band energies calculated from DFT and DFT + D are found to be same in both the compounds. This is may be due to the fact that as pressure increases the overlapping of $\mathrm{N}-\mathrm{p}$ orbitals increases which in turn diminishes the dispersion forces between the azide ion layers. The pressure dependence of the band gap values of $\mathrm{LiN}_{3}$ and $\mathrm{KN}_{3}$ calculated through the DFT and DFT $+\mathrm{D}$ are presented in figure $8 \mathrm{a}$ and $\mathrm{b}$. The magnitude of the band gap is decreasing monotonically as pressure increases in both the compounds implying the possibility of these compounds becoming semiconductors at high pressures. This is a significant feature in achieving the polymeric nitrogen which is found to be semiconductor in nature. $^{8}$

\subsection{Total and partial density of states (DOS) of $\mathrm{LiN}_{3}$ and $\mathrm{KN}_{3}$}

In figure $9 \mathrm{a}$ and $\mathrm{b}$ we have shown the calculated total and partial DOS of $\mathrm{LiN}_{3}$ and $\mathrm{KN}_{3}$ within the DFT+D

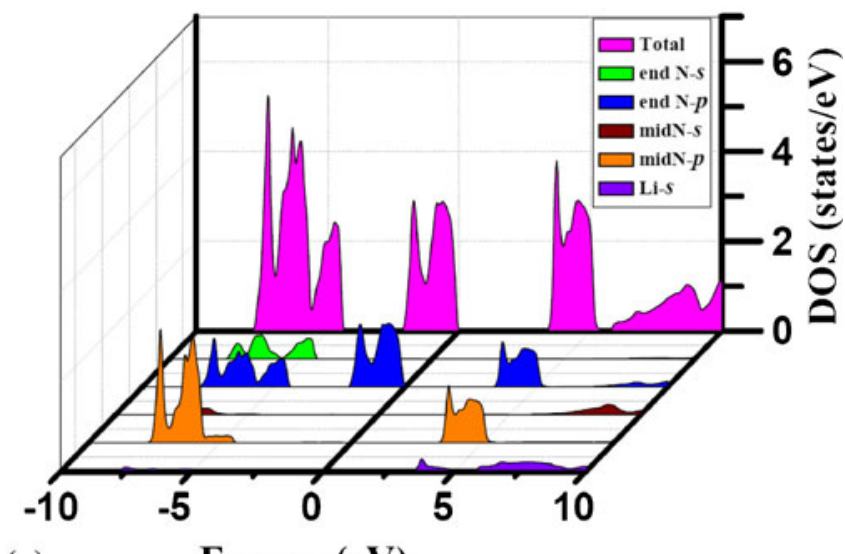

(a) Energy (eV)

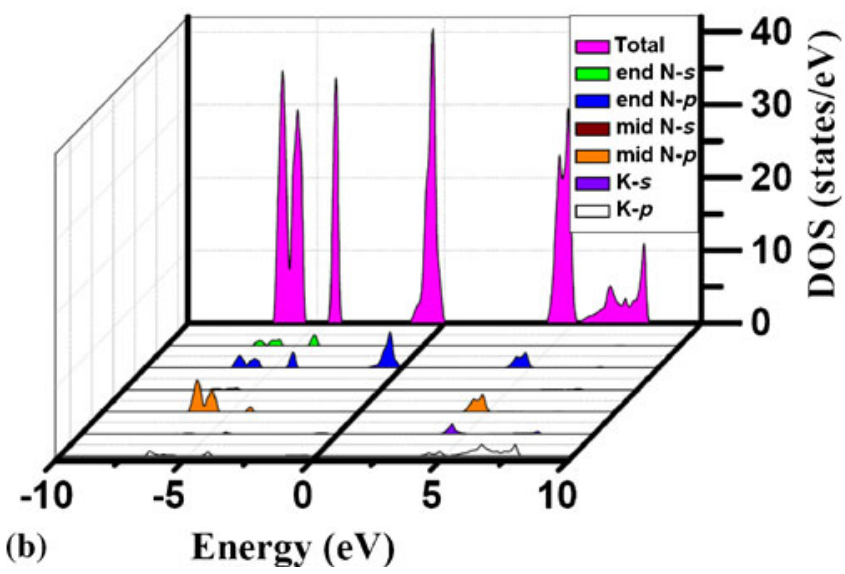

Figure 9. (a) The total and partial density of states of $\mathrm{LiN}_{3}$ and (b) the total and partial density of states of $\mathrm{KN}_{3}$ calculated at ambient pressure. 
method. In both the compounds, the states in valence band including the bands at the Fermi level are entirely due to the p-states of $\mathrm{N}$ atoms and those in conduction band are from the s-states of metal atom and p-states of $\mathrm{N}$ atoms, respectively. In detail, the states at the Fermi level are due to p-states of end $\mathrm{N}$ atom whereas the states lying at $-5 \mathrm{eV}$ are due to the p-states of mid $\mathrm{N}$ atom. The metal atom states do not play much role in the valence band indicating that in both the compounds ionic bonding is dominating, which is a well-known feature of the materials having a positive cation and a negative anion similar to that of known alkali halides, a well-known ionic compounds. As pressure increases, the states of end $\mathrm{N}$ and mid $\mathrm{N}$ are delocalizing as shown in figure 10a and $\mathrm{b}$. One can also notice that the width of the energy interval of p-states increases in the valence band. This is due to the fact that as pressure increases the intra molecular interactions between the $\mathrm{N}$ atoms of the azide ion enhances and therefore the states become broadened. A significant feature of the calculated DOS under pressure is that the contribution of metal atom states in the conduction band increases. Over all, from the study of DOS of $\mathrm{LiN}_{3}$ and $\mathrm{KN}_{3}$ we came to the

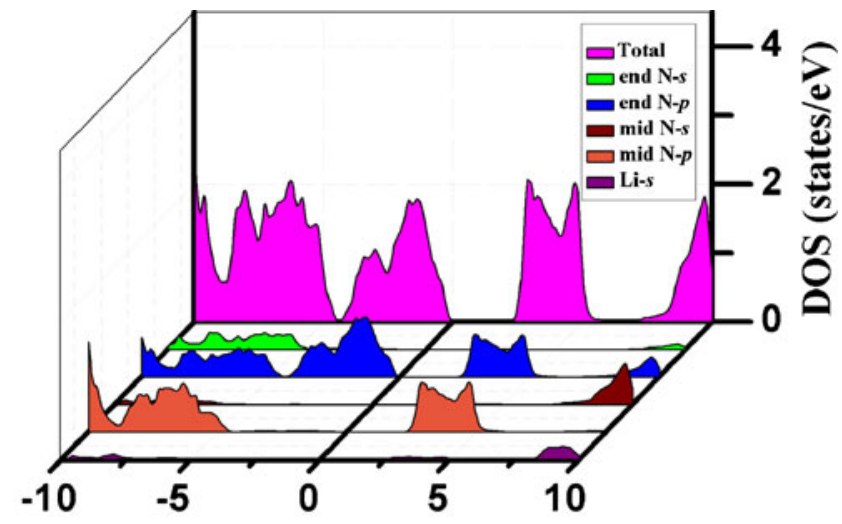

(a) Energy (eV)

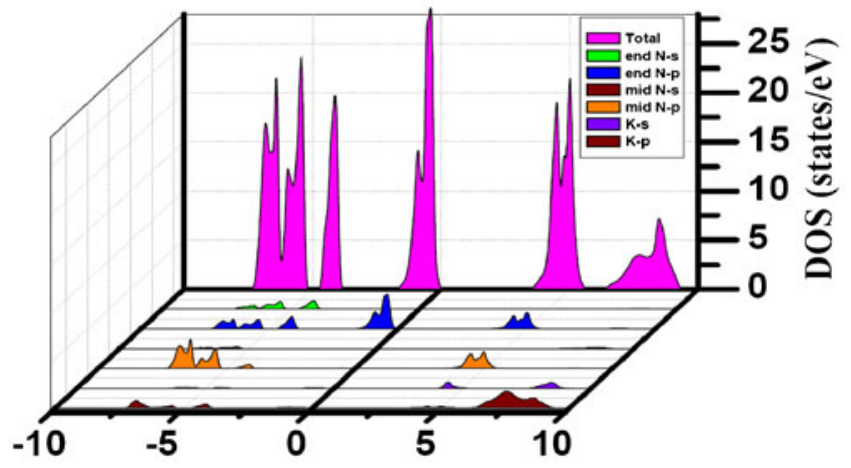

(b)

Energy (eV)

Figure 10. (a) The total and partial density of states of $\mathrm{LiN}_{3}$ calculated at $60 \mathrm{GPa}$ and (b) the total and partial density of states of $\mathrm{KN}_{3}$ calculated at pressure of $16 \mathrm{GPa}$. conclusion that the azide ion plays a major role in bonding properties of the alkali metal azides.

\section{Conclusions}

In conclusion, we have studied the high pressure behaviour of monoclinic $\mathrm{LiN}_{3}$ and tetragonal $\mathrm{KN}_{3}$ molecular crystals using density functional theory including van der Waals interactions. The calculated ground state properties are greatly improved by the DFT +D method over the usual DFT results. The calculated $\mathrm{P}-\mathrm{V}$ relation for both the compounds is wellreproduced by calculations with the DFT $+\mathrm{D}$ method. Our calculations clearly show that the compressibility of the materials is anisotropic which is also consistent with experiment. The calculated electronic band structure of $\mathrm{LiN}_{3}$ and $\mathrm{KN}_{3}$ clearly shows that both the compounds are direct band gap insulators. As pressure increases, the band gap decreases implying the possibility to become semiconductor which is a precursor for the formation of polymeric nitrogen. The total and partial density of states of $\mathrm{LiN}_{3}$ and $\mathrm{KN}_{3}$ are also supporting this fact. As pressure increases, the states become broadened, implying the delocalization of the azide ion states which is also a witness towards the formation of polymeric nitrogen. We also found that at high pressures, the dispersion interactions do not play much role in these compounds.

\section{Acknowledgements}

KRB thanks the Defense Research and Development Organization (DRDO) for funding through Advanced Centre of Research in High Energy Materials (ACRHEM) and Centre for Modelling Simulation and Design (CMSD), University of Hyderabad for providing computational facilities.

\section{References}

1. Evans B L, Yoffe A D and Gray P 1959 Chem. Rev. 59 515

2. Fair H D and Walke R F 1977 Energetic materilas Vol. 1 New York: Plenum Press

3. Seel M and Kunz A B, 1991 Int. J. Quantum. Chem. 39 149

4. Younk E H and Kunz A B 1997 Int. J. Quantum Chem. 63615

5. Pringle G E and Noakers D E 1968 Acta Crystallogr. B24 262

6. Muller U Z 1972 Z. Anorg. Allg. Chem. 392159

7. Eremets M I, Hemley R J, Mao H K and Gregoryanz E 2001 Nature 411170

8. Eremets M I, Gavriliuk A G, Trojan I A, Dzivenko D A and Boehler R 2004 Nat. Mat. 3558 
9. Eremets M I, Popov M Yu, Trojan I A, Denisov V N, Boehler R and Hemley R J 2004 J. Chem. Phys. 120 10618

10. Medvedev S A, Trojan I A, Eremets M I, Palasyuk T, Klapotke T M and Evers J 2009 J. Phys. Condens. Mat. 21195404

11. Hou D, Zhang F, Cheng J, Hannon T, Zhu H, Wu J and Ma Y 2011 Phys. Rev. B. 84064127

12. Ji C, Zhang F, Hou D, Zhu H, Wu J, Chyu M-C, Levitas V I, Maa Y 2011 J. Phys. Chem. Solids 72736

13. Ramesh B K, Bheema L Ch, Tewari Surya P, Vaitheeswaran G 2011 J. Phys. Chem. A. 1154521
14. Segall M, Lindan P, Probert M, Pickard C, Hasnip P, Clark S and Payne M J 2002 J. Phys. Condens. Mat. 14271

15. Perdew J P, Burke K and Ernzerhof M 1996 Phys. Rev. Lett. 773865

16. Grimme S 2006 J. Comp. Chem. 271787

17. Vanderbilt D 1990 Phys. Rev. B. 417892

18. Monkhorst H J and Pack J 1976 Phys. Rev. B. 135188

19. Perger W F 2010 Int. J. Quantum Chem. 1101916

20. Zhu W, Xiao J and Xiao H 2006 Chem. Phys. Lett. 422 117

21. Jones R O and Gunnarsson O 1989 Rev. Mod. Phys. 61 689-746 